International Journal of Applied Mathematics

Volume 33 No. $5 \quad 2020,879-901$

ISSN: 1311-1728 (printed version); ISSN: 1314-8060 (on-line version)

doi: http://dx.doi.org/10.12732/ijam.v33i5.10

\title{
MODIFIED POPOV'S SUBGRADIENT EXTRAGRADIENT ALGORITHM WITH INERTIAL TECHNIQUE FOR EQUILIBRIUM PROBLEMS AND ITS APPLICATIONS
}

\author{
Nopparat Wairojjana ${ }^{1}$, Habib ur Rehman ${ }^{2}$, \\ Nuttapol Pakkaranang ${ }^{2}$, and Tiwabhorn Khanpanuk ${ }^{3} \S$ \\ ${ }^{1}$ Applied Mathematics Program, Fac. of Science and \\ Technology, Valaya Alongkorn Rajabhat University \\ under the Royal Patronage \\ Pathumthani, 13180, THAILAND \\ 2 Dept. of Mathematics, Fac. of Science \\ King Mongkut's University of Technology Thonburi \\ Bangkok 10140, THAILAND \\ ${ }^{3}$ Dept. of Mathematics, Fac. of Science and Technology \\ Phetchabun Rajabhat University \\ Phetchabun 67000, THAILAND
}

\begin{abstract}
In this paper, we are introducing a new algorithm that is based on a subgradient and an inertial scheme using an explicit method for step size evaluation to solve pseudomonotone equilibrium problems. The weak convergence theorem for an algorithm is well established on the basis of standard cost bifunction conditions. A useful feature of a method that it operates without a line search procedure or prior Lipschitz-type constant information. The reason for this is that it has used a step size rule that is modified for each iteration on the basis of some of the previous iterations. For computational experiment, we consider the well-known Nash-Cournot equilibrium model to support our well-established convergence result and to see that our suggested methodology has a competitive edge over existing ones.
\end{abstract}

AMS Subject Classification: 65Y05, 65K15, 47H05, 47H10

Received: July 27, 2020

(C) 2020 Academic Publications

${ }^{\S}$ Correspondence author 
Key Words: pseudomonotone equilibrium problems; weak convergence theorem; Lipschitz-type conditions; variational inequality problems

\section{Introduction}

Equilibrium problem (EP) had many mathematical problems as a particular case, such as the variational inequality problems (VIP), problem of optimization, fixed point problems, complementarity problems, Nash equilibrium of non-cooperative games, the saddle point and vector minimization problems (for more details see e.g., $[1,2,3]$ ). To the best of our knowledge, the term "equilibrium problem" in an individual way presented in 1992 by Muu and Oettli [4] and has been further extended by Blum and Oettli [1]. The problem of equilibrium is also known as the famous Ky Fan inequality [5]. One of the most interesting and effective areas of research in equilibrium problem theory is the development of new iterative methods, the improvement of existing methods, and the examination of their convergence analysis. Several methods have already been used in recent years to estimate the solution of the problem of equilibrium in both finite and infinite-dimensional spaces, i.e., the extragradient methods $[6,7,8,9,9,10,11,12,13,14,15,16]$ and others in $[17,18,19,20,21,22,23,24,25,26]$.

The Proximal point method (PPM) is one of the well-established methods for studying numerical equilibrium problems. Martinet [27] originally introduced this method for monotone variational inequalities problems, which was eventually extended to monotone operators by Rockafellar [28]. In addition, Mudafi [29] extended the proximal point method to solve the problem of equilibrium involving monotone bifunction. The proximal point method is usually used to solve monotone equilibrium problems. Then, every regularized subproblem turns into a strongly monotone equilibrium problem, and a unique solution exists. On the other hand, another well-known technique is the auxiliary problem principle, which is based on the idea of creating a new, identical problem that is easier to solve than the original problem. This concept was first studied by Cohen [30] to solve optimization problems and was eventually used to solve variational inequality problems [31]. As an extension, Mastroeni [32] studied the auxiliary problem principle in the case of strongly monotone equilibrium problems.

In 2018, Liu et al. [33] proposed a modification of the Popov's extragradient method [34] to solve pseudomonotone equilibrium problems in real Hilbert space. It is mandatory to solve two minimization problems on a closed convex 
set for each iteration to generate an iterative sequence due to the method in [33] and appropriate fixed step size is required to solve each minimization problem. Liu et al. [33] iterative sequence $\left\{x_{n}\right\}$ describes as follows: Let $x_{0}, y_{0} \in \mathbb{E}$ and $0<\lambda \leq \frac{1}{2 c_{2}+4 c_{1}}$. Set

(i)

$$
\left\{\begin{array}{c}
x_{1}=\underset{y \in \mathbb{K}}{\arg \min }\left\{\lambda f\left(y_{0}, y\right)+\frac{1}{2}\left\|x_{0}-y\right\|^{2}\right\}, \\
y_{1}=\underset{y \in \mathbb{K}}{\arg \min }\left\{\lambda f\left(y_{0}, y\right)+\frac{1}{2}\left\|x_{1}-y\right\|^{2}\right\} .
\end{array}\right.
$$

(ii) For $x_{n}, y_{n}$ and $y_{n-1}$, construct a half-space

$$
E_{n}=\left\{z \in \mathbb{E}:\left\langle x_{n}-\lambda_{n} v_{n-1}-y_{n}, z-y_{n}\right\rangle \leq 0\right\}
$$

where $v_{n-1} \in \partial_{2} f\left(y_{n-1}, y_{n}\right)$. Compute

$$
\left\{\begin{array}{l}
x_{n+1}=\underset{y \in E_{n}}{\arg \min }\left\{\lambda f\left(y_{n}, y\right)+\frac{1}{2}\left\|x_{n}-y\right\|^{2}\right\}, \\
y_{n+1}=\underset{y \in \mathbb{K}}{\arg \min }\left\{\lambda f\left(y_{n}, y\right)+\frac{1}{2}\left\|x_{n+1}-y\right\|^{2}\right\} .
\end{array}\right.
$$

The aim of this study is to modify the Algorithm 3.1 in [33] by incorporating the results in [15] to solve a class of equilibrium problems involving pseudomonotone bifunction. This method also includes the inertial term introduced by Polyak [35] with Algorithm 3.1 in [33], which is used to improve the iterative sequence of the solution required. The key feature of such algorithms is that they are independent of any line search method and there is no need to have previous information about Lipschitz-type constants. Instead, they apply the step size rule that is revised for each iteration on the basis of certain previous iterations. The weak convergence of the corresponding method is demonstrated on the basis of standard assumptions concerning the cost bifunction.

This paper is arranged in the following way: Section 2 there are some definitions and basic results that will be used in this article. Section 3 defines and provides the convergence theorem of an inertial-type algorithm involving a pseudomonotone bifunction. Section 4 set out some application of our results. Section 5 sets out experimental studies to demonstrate the algorithmic performance on tests of a problem modelled on the Nash-Cournot equilibrium model. 


\section{Background}

Let $\mathbb{K} \subset \mathbb{E}$ be a convex and closed set of a real Hilbert space $\mathbb{E}$. The inner product is denoted by $\langle.,$.$\rangle and the induced norm is denoted by \|$.$\| . Let f$ be a bifunction $f: \mathbb{E} \times \mathbb{E} \rightarrow \mathbb{R}$ with $E P(f, \mathbb{K})$ denote the solution set of an equilibrium problem over $\mathbb{K}$ and $p^{*}$ is any element of $E P(f, \mathbb{K})$.

Let consider the following definitions of a monotonicity of a bifunction (see $[1,36]$ for details). A bifunction $f: \mathbb{E} \times \mathbb{E} \rightarrow \mathbb{R}$ on $\mathbb{K}$ for $\gamma>0$ is said to be

(1) strongly monotone if

$$
f(\breve{x}, \breve{y})+f(\breve{y}, \breve{x}) \leq-\gamma\|\breve{x}-\breve{y}\|^{2}, \forall \breve{x}, \breve{y} \in \mathbb{K}
$$

(2) monotone if

$$
f(\breve{x}, \breve{y})+f(\breve{y}, \breve{x}) \leq 0, \forall \breve{x}, \breve{y} \in \mathbb{K}
$$

(3) strongly pseudomonotone if

$$
f(\breve{x}, \breve{y}) \geq 0 \Longrightarrow f(\breve{y}, \breve{x}) \leq-\gamma\|\breve{x}-\breve{y}\|^{2}, \forall \breve{x}, \breve{y} \in \mathbb{K} ;
$$

(4) pseudomonotone if

$$
f(\breve{x}, \breve{y}) \geq 0 \Longrightarrow f(\breve{y}, \breve{x}) \leq 0, \forall \breve{x}, \breve{y} \in \mathbb{K} ;
$$

(5) satisfying the Lipschitz-type condition on $\mathbb{K}$ if there exits two numbers $c_{1}, c_{2}>0$, such that

$$
f(\breve{x}, \breve{z})-c_{1}\|\breve{x}-\breve{y}\|^{2}-c_{2}\|\breve{y}-\breve{z}\|^{2} \leq f(\breve{x}, \breve{y})+f(\breve{y}, \breve{z}), \forall \breve{x}, \breve{y}, \breve{z} \in \mathbb{K} .
$$

Note: We have the following consequences from the above definitions:

$$
(1) \Longrightarrow(2) \Longrightarrow(4) \text { and } \quad(1) \Longrightarrow(3) \Longrightarrow(4) \text {. }
$$

For given $\mathbb{K}$ to be a nonempty closed and convex subset of a real Hilbert space $\mathbb{E}$ and let $f: \mathbb{E} \times \mathbb{E} \rightarrow \mathbb{R}$ be a bifunction through $f(\breve{x}, \breve{x})=0$ for every $\breve{x} \in \mathbb{K}$. The equilibrium problem $[1,5]$ for $f$ over $\mathbb{K}$ is defined as follows:

$$
\text { Find } p^{*} \in \mathbb{K} \text { such that } f\left(p^{*}, \breve{y}\right) \geq 0, \forall \breve{y} \in \mathbb{K} \text {. }
$$

Let $g: \mathbb{K} \rightarrow \mathbb{R}$ is a convex function and subdifferential of $g$ at $\breve{x} \in \mathbb{K}$ is defined by

$$
\partial g(\breve{x})=\{w \in \mathbb{K}: g(\breve{y})-g(\breve{x}) \geq\langle w, \breve{y}-\breve{x}\rangle, \forall \breve{y} \in \mathbb{K}\}
$$


A normal cone of $\mathbb{K}$ at $\breve{x} \in \mathbb{K}$ is defined by

$$
N_{\mathbb{K}}(\breve{x})=\{w \in \mathbb{E}:\langle w, \breve{y}-\breve{x}\rangle \leq 0, \forall \breve{y} \in \mathbb{K}\}
$$

A projection $P_{\mathbb{K}}(\breve{x})$ of $\breve{x}$ onto a closed, convex subset $\mathbb{K}$ of $\mathbb{E}$ is defined by

$$
P_{\mathbb{K}}(\breve{x})=\underset{\breve{y} \in \mathbb{K}}{\arg \min }\{\|\breve{y}-\breve{x}\|\}
$$

Lemma 2.1. ([37]) Assume $\mathbb{K}$ be a non-empty, closed and convex subset of a real Hilbert space $\mathbb{E}$ and $g: \mathbb{K} \rightarrow \mathbb{R}$ be a convex, subdifferentiable and lower semicontinuous function on $\mathbb{K}$. Then, $\breve{p} \in \mathbb{K}$ is a minimizer of a function $g$ if and only if $0 \in \partial g(\breve{p})+N_{\mathbb{K}}(\breve{p})$, where $\partial g(\breve{p})$ and $N_{\mathbb{K}}(\breve{p})$ denotes the subdifferential of $g$ at $\breve{p}$ and the normal cone of $\mathbb{K}$ at $\breve{p}$, respectively.

Lemma 2.2. ([38]) For $\breve{x}, \breve{y} \in \mathbb{E}$ and $ð \in \mathbb{R}$, then the following relationship holds:

$$
\|ð \breve{x}+(1-ð) \breve{y}\|^{2}=ð\|\breve{x}\|^{2}+(1-ð)\|\breve{y}\|^{2}-ð(1-ð)\|\breve{x}-\breve{y}\|^{2}
$$

Lemma 2.3. ([39]) Let $a_{n}, b_{n}$ and $c_{n}$ are sequences in $[0,+\infty)$ and

$$
a_{n+1} \leq a_{n}+b_{n}\left(a_{n}-a_{n-1}\right)+c_{n}, \forall n \geq 1, \text { with } \sum_{n=1}^{+\infty} c_{n}<+\infty
$$

with $b>0$ and $0 \leq b_{n} \leq b<1 \forall n \in \mathbb{N}$. Then, the following relations are established.

(i) $\sum_{n=1}^{+\infty}\left[a_{n}-a_{n-1}\right]_{+}<\infty$, with $[s]_{+}:=\max \{s, 0\}$;

(ii) $\lim _{n \rightarrow+\infty} a_{n}=a^{*} \in[0, \infty)$.

Lemma 2.4. ([40]) labelopial Let $\left\{\xi_{n}\right\}$ be a sequence in $\mathbb{E}$ and $\mathbb{K} \subset \mathbb{E}$ such that

(i) For each $\xi \in \mathbb{K}, \lim _{n \rightarrow \infty}\left\|\xi_{n}-\xi\right\|$ exists;

(ii) all sequentially weak cluster point of $\left\{\xi_{n}\right\}$ lies in $\mathbb{K}$.

Then, $\left\{\xi_{n}\right\}$ weakly converges to a point in $\mathbb{K}$. 
Assumption 2.1. Let $f: \mathbb{E} \times \mathbb{E} \rightarrow \mathbb{R}$ satisfies the following conditions:

(a1) $f(\breve{z}, \breve{z})=0, \forall \breve{z} \in \mathbb{K}$ and $f$ is pseudomonotone on $\mathbb{K}$;

(a2) $f$ is Lipschitz-type continuous on $\mathbb{E}$ with $c_{1}, c_{2}>0$;

(a3) $\limsup _{n \rightarrow \infty} f\left(z_{n}, z\right) \leq f(\breve{z}, z)$ for $z \in \mathbb{K}$ and satisfies $z_{n} \rightarrow \breve{z}$;

(a4) $f(\breve{z},$.$) is convex and subdifferentiable on \mathbb{K}$ for $\breve{z} \in \mathbb{K}$.

\section{Main Results}

In this section, we have set up our main method to solve the problem of pseudomonotone (EP) containing a bi-functional Lipschitz-type condition. It involves a certain step size rule and an inertial approach to improve the performance of the iterative sequence. A detailed methodology is provided in next page.

Lemma 3.1. Let $f: \mathbb{E} \times \mathbb{E} \rightarrow \mathbb{R}$ satisfies the conditions (a1)-(a4). Then, for $p^{*} \in E P(f, \mathbb{K}) \neq \emptyset$, we have

$$
\begin{aligned}
\left\|z_{n}-p^{*}\right\|^{2} \leq & \left\|w_{n}-p^{*}\right\|^{2}-\left(1-\frac{2 \mu \lambda_{n}}{\lambda_{n+1}}\right)\left\|w_{n}-y_{n}\right\|^{2} \\
& -\left(1-\frac{\mu \lambda_{n}}{\lambda_{n+1}}\right)\left\|z_{n}-y_{n}\right\|^{2}+\frac{2 \mu \lambda_{n}}{\lambda_{n+1}}\left\|w_{n}-y_{n-1}\right\|^{2} .
\end{aligned}
$$

Proof. The value of $z_{n}$ gives that

$$
0 \in \partial_{2}\left\{\lambda_{n} f\left(y_{n}, y\right)+\frac{1}{2}\left\|w_{n}-y\right\|^{2}\right\}\left(z_{n}\right)+N_{E_{n}}\left(z_{n}\right) .
$$

For $\omega \in \partial_{2} f\left(y_{n}, z_{n}\right)$ and $\bar{\omega} \in N_{E_{n}}\left(z_{n}\right)$ such that $\lambda_{n} \omega+z_{n}-w_{n}+\bar{\omega}=0$. Thus, we have

$$
\left\langle w_{n}-z_{n}, y-z_{n}\right\rangle=\lambda_{n}\left\langle\omega, y-z_{n}\right\rangle+\left\langle\bar{\omega}, y-z_{n}\right\rangle, \forall y \in E_{n} .
$$

Since $\bar{\omega} \in N_{E_{n}}\left(z_{n}\right)$ then $\left\langle\bar{\omega}, y-z_{n}\right\rangle \leq 0$, for all $y \in E_{n}$. Thus,

$$
\lambda_{n}\left\langle\omega, y-z_{n}\right\rangle \geq\left\langle w_{n}-z_{n}, y-z_{n}\right\rangle, \forall y \in E_{n} .
$$




\section{Algorithm 1}

INITIALIZATION: Let $x_{-1}, x_{0}, y_{0} \in \mathbb{E}, \lambda_{0}=\lambda_{1}>0$ and sequence $\vartheta_{n}$ is nondecreasing and satisfying $0 \leq \vartheta_{n}<\vartheta<\sqrt{5}-2$. Set

$$
\begin{aligned}
& x_{1}=\arg \min \left\{\lambda_{0} f\left(y_{0}, y\right)+\frac{1}{2}\left\|w_{0}-y\right\|^{2}: y \in \mathbb{K}\right\}, \\
& y_{1}=\arg \min \left\{\lambda_{0} f\left(y_{0}, y\right)+\frac{1}{2}\left\|w_{1}-y\right\|^{2}: y \in \mathbb{K}\right\},
\end{aligned}
$$

where $w_{0}=x_{0}+\vartheta_{0}\left(x_{0}-x_{-1}\right)$ and $w_{1}=x_{1}+\vartheta_{1}\left(x_{1}-x_{0}\right)$.

Iterative STEPS: Given $x_{n-1}, y_{n-1}, x_{n}, y_{n}$ for $n \geq 1$. Determine a set $E_{n}=\left\{z \in \mathbb{E}:\left\langle w_{n}-\lambda_{n} v_{n-1}-y_{n}, z-y_{n}\right\rangle \leq 0\right\}$, where $v_{n-1} \in \partial_{2} f\left(y_{n-1}, y_{n}\right)$. STEP 1: Compute

$$
x_{n+1}=\left(1-\beta_{n}\right) w_{n}+\beta_{n} z_{n},
$$

where $z_{n}=\arg \min \left\{\lambda_{n} f\left(y_{n}, y\right)+\frac{1}{2}\left\|w_{n}-y\right\|^{2}: y \in E_{n}\right\}$ and $w_{n}=x_{n}+\vartheta_{n}\left(x_{n}-x_{n-1}\right)$ and nonincreasing $0<\beta \leq \beta_{n} \leq 1$.

STEP 2: Assume $\mu(\vartheta)>0$ and set $d=f\left(y_{n-1}, z_{n}\right)-f\left(y_{n-1}, y_{n}\right)-f\left(y_{n}, z_{n}\right)$ such that

$$
\lambda_{n+1}=\left\{\begin{array}{lc}
\min \left\{\lambda_{n}, \frac{\mu\left(\left\|y_{n-1}-y_{n}\right\|^{2}+\left\|y_{n}-z_{n}\right\|^{2}\right)}{2 d}\right\} & \text { if } \quad \begin{array}{c}
d>0, \\
\lambda_{n}
\end{array} \text { else. }
\end{array}\right.
$$

SteP 3: Compute

$$
y_{n+1}=\arg \min \left\{\lambda_{n+1} f\left(y_{n}, y\right)+\frac{1}{2}\left\|w_{n+1}-y\right\|^{2}: y \in \mathbb{K}\right\},
$$

where $w_{n+1}=x_{n+1}+\vartheta_{n+1}\left(x_{n+1}-x_{n}\right)$.

STEP 4: If $z_{n}=w_{n}=y_{n}$ then stop, otherwise set $n:=n+1$ and go back to Step 1. 
Due to $\omega \in \partial f\left(y_{n}, z_{n}\right)$, we have

$$
f\left(y_{n}, y\right)-f\left(y_{n}, z_{n}\right) \geq\left\langle\omega, y-z_{n}\right\rangle, \forall y \in \mathbb{E} .
$$

From (1) and (2), we obtain

$$
\lambda_{n} f\left(y_{n}, y\right)-\lambda_{n} f\left(y_{n}, z_{n}\right) \geq\left\langle w_{n}-z_{n}, y-z_{n}\right\rangle, \forall y \in E_{n} .
$$

Similarly to (3) and substituting $y=z_{n}$, we have

$$
\lambda_{n}\left\{f\left(y_{n-1}, z_{n}\right)-f\left(y_{n-1}, y_{n}\right)\right\} \geq\left\langle w_{n}-y_{n}, z_{n}-y_{n}\right\rangle .
$$

Substituting $y=p^{*}$ into (3) such that

$$
\lambda_{n} f\left(y_{n}, p^{*}\right)-\lambda_{n} f\left(y_{n}, z_{n}\right) \geq\left\langle w_{n}-z_{n}, p^{*}-z_{n}\right\rangle .
$$

Since $f\left(p^{*}, y_{n}\right) \geq 0$ and from given $f\left(y_{n}, p^{*}\right) \leq 0$ implies that

$$
\left\langle w_{n}-z_{n}, z_{n}-p^{*}\right\rangle \geq \lambda_{n} f\left(y_{n}, z_{n}\right) .
$$

By value of $\lambda_{n+1}$ we get

$$
f\left(y_{n-1}, z_{n}\right)-f\left(y_{n-1}, y_{n}\right)-f\left(y_{n}, z_{n}\right) \leq \frac{\mu\left(\left\|y_{n-1}-y_{n}\right\|^{2}+\left\|z_{n}-y_{n}\right\|^{2}\right)}{2 \lambda_{n+1}}
$$

which, after multiplying both sides by $\lambda_{n}>0$, implies that

$$
\begin{aligned}
\lambda_{n} f\left(y_{n}, z_{n}\right) \geq & \lambda_{n} f\left(y_{n-1}, z_{n}\right)-\lambda_{n} f\left(y_{n-1}, y_{n}\right) \\
& -\frac{\lambda_{n} \mu\left(\left\|y_{n-1}-y_{n}\right\|^{2}+\left\|z_{n}-y_{n}\right\|^{2}\right)}{2 \lambda_{n+1}} .
\end{aligned}
$$

Combining (6) and (7) such that

$$
\begin{aligned}
\left\langle w_{n}-z_{n}, z_{n}-p^{*}\right\rangle \geq & \lambda_{n}\left\{f\left(y_{n-1}, z_{n}\right)-f\left(y_{n-1}, y_{n}\right)\right\} \\
& -\frac{\mu \lambda_{n}}{2 \lambda_{n+1}}\left\|y_{n-1}-y_{n}\right\|^{2}-\frac{\mu \lambda_{n}}{2 \lambda_{n+1}}\left\|z_{n}-y_{n}\right\|^{2} .
\end{aligned}
$$

Combining (4) and (8) such that

$$
\begin{aligned}
\left\langle w_{n}-z_{n}, z_{n}-p^{*}\right\rangle \geq & \left\langle w_{n}-y_{n}, z_{n}-y_{n}\right\rangle \\
& -\frac{\mu \lambda_{n}}{2 \lambda_{n+1}}\left\|y_{n-1}-y_{n}\right\|^{2}-\frac{\mu \lambda_{n}}{2 \lambda_{n+1}}\left\|z_{n}-y_{n}\right\|^{2} .
\end{aligned}
$$


We have the following formulas as follows:

$$
\begin{gathered}
-2\left\langle w_{n}-z_{n}, z_{n}-p^{*}\right\rangle=-\left\|w_{n}-p^{*}\right\|^{2}+\left\|z_{n}-w_{n}\right\|^{2}+\left\|z_{n}-p^{*}\right\|^{2} \\
2\left\langle y_{n}-w_{n}, y_{n}-z_{n}\right\rangle=\left\|w_{n}-y_{n}\right\|^{2}+\left\|z_{n}-y_{n}\right\|^{2}-\left\|w_{n}-z_{n}\right\|^{2}
\end{gathered}
$$

and

$\left\|y_{n-1}-y_{n}\right\|^{2} \leq\left(\left\|y_{n-1}-w_{n}\right\|+\left\|w_{n}-y_{n}\right\|\right)^{2} \leq 2\left\|y_{n-1}-w_{n}\right\|^{2}+2\left\|w_{n}-y_{n}\right\|^{2}$

Combing above facts and (9), completes the proof.

Theorem 3.1. Let $\left\{w_{n}\right\},\left\{y_{n}\right\}$ and $\left\{x_{n}\right\}$ generated by Algorithm 1 converge weakly to the solution $p^{*}$ and

$$
0<\mu<\frac{\frac{1}{2}-2 \vartheta-\frac{1}{2} \vartheta^{2}}{2-\vartheta+2 \vartheta^{2}+\vartheta^{3}} \quad \text { and } \quad 0 \leq \vartheta_{n} \leq \vartheta<\sqrt{5}-2 .
$$

Proof. By value of $x_{n+1}$, we have

$$
\begin{aligned}
\left\|x_{n+1}-p^{*}\right\|^{2}= & \left\|\left(1-\beta_{n}\right)\left(w_{n}-p^{*}\right)+\beta_{n}\left(z_{n}-p^{*}\right)\right\|^{2} \\
\leq & \left(1-\beta_{n}\right)\left\|w_{n}-p^{*}\right\|^{2}+\beta_{n}\left\|z_{n}-p^{*}\right\|^{2} . \\
= & \left\|w_{n}-p^{*}\right\|^{2}-\beta_{n}\left(1-\frac{2 \mu \lambda_{n}}{\lambda_{n+1}}\right)\left\|w_{n}-y_{n}\right\|^{2} \\
& -\beta_{n}\left(1-\frac{\mu \lambda_{n}}{\lambda_{n+1}}\right)\left\|z_{n}-y_{n}\right\|^{2}+\frac{2 \mu \lambda_{n}}{\lambda_{n+1}} \beta_{n}\left\|w_{n}-y_{n-1}\right\|^{2} .
\end{aligned}
$$

By using $w_{n}$ and Lemma 2.2, we obtain

$$
\begin{aligned}
& \left\|w_{n}-p^{*}\right\|^{2} \\
& =\left\|\left(1+\vartheta_{n}\right)\left(x_{n}-p^{*}\right)-\vartheta_{n}\left(x_{n-1}-p^{*}\right)\right\|^{2} \\
& =\left(1+\vartheta_{n+1}\right)\left\|x_{n}-p^{*}\right\|^{2}-\vartheta_{n}\left\|x_{n-1}-p^{*}\right\|^{2}+\vartheta(1+\vartheta)\left\|x_{n}-x_{n-1}\right\|^{2} .
\end{aligned}
$$

Combining (10) and (11), we get

$$
\begin{aligned}
& \left\|x_{n+1}-p^{*}\right\|^{2}+\frac{2 \mu \beta_{n+1} \lambda_{n+1}}{\lambda_{n+2}}\left\|w_{n+1}-y_{n}\right\|^{2} \\
& \leq\left(1+\vartheta_{n+1}\right)\left\|x_{n}-p^{*}\right\|^{2}-\vartheta_{n}\left\|x_{n-1}-p^{*}\right\|^{2}+\vartheta(1+\vartheta)\left\|x_{n}-x_{n-1}\right\|^{2} \\
& \quad+\frac{2 \mu \beta_{n} \lambda_{n}}{\lambda_{n+1}}\left\|w_{n}-y_{n-1}\right\|^{2}-\beta_{n}\left(1-\frac{2 \mu \lambda_{n}}{\lambda_{n+1}}\right)\left\|w_{n}-y_{n}\right\|^{2}
\end{aligned}
$$




$$
-\beta_{n}\left(1-\frac{\mu \lambda_{n}}{\lambda_{n+1}}\right)\left\|z_{n}-y_{n}\right\|^{2}+\frac{2 \mu \beta_{n} \lambda_{n+1}}{\lambda_{n+2}}\left\|w_{n+1}-y_{n}\right\|^{2}
$$

By $x_{n+1}$ and Lemma 2.2, we obtain

$$
\begin{aligned}
& \left\|x_{n+1}-y_{n}\right\|^{2} \\
& =\left\|\left(1-\beta_{n}\right)\left(w_{n}-y_{n}\right)+\beta_{n}\left(z_{n}-y_{n}\right)\right\|^{2} \\
& \leq\left(1-\beta_{n}\right)\left\|w_{n}-y_{n}\right\|^{2}+\beta_{n}\left\|z_{n}-y_{n}\right\|^{2} \leq\left\|w_{n}-y_{n}\right\|^{2}+\left\|z_{n}-y_{n}\right\|^{2} .
\end{aligned}
$$

By $w_{n+1}$ through Lemma 2.2, we obtain

$$
\begin{aligned}
& \left\|w_{n+1}-y_{n}\right\|^{2} \\
& =\left\|x_{n+1}+\vartheta_{n+1}\left(x_{n+1}-x_{n}\right)-y_{n}\right\|^{2} \\
& \leq\left(1+\vartheta_{n+1}\right)\left\|x_{n+1}-y_{n}\right\|^{2}+\vartheta_{n+1}\left(1+\vartheta_{n+1}\right)\left\|x_{n+1}-x_{n}\right\|^{2} \\
& \leq(1+\vartheta)\left[\left\|w_{n}-y_{n}\right\|^{2}+\left\|z_{n}-y_{n}\right\|^{2}\right]+\vartheta(1+\vartheta)\left\|x_{n+1}-x_{n}\right\|^{2} .
\end{aligned}
$$

Combining (12) and (14), we obtain

$$
\begin{aligned}
& \left\|x_{n+1}-p^{*}\right\|^{2}+\frac{2 \mu \beta_{n+1} \lambda_{n+1}}{\lambda_{n+2}}\left\|w_{n+1}-y_{n}\right\|^{2} \\
& \leq \quad\left(1+\vartheta_{n+1}\right)\left\|x_{n}-p^{*}\right\|^{2}-\vartheta_{n}\left\|x_{n-1}-p^{*}\right\|^{2}+\vartheta(1+\vartheta)\left\|x_{n}-x_{n-1}\right\|^{2} \\
& \quad+\frac{2 \mu \beta_{n} \lambda_{n}}{\lambda_{n+1}}\left\|w_{n}-y_{n-1}\right\|^{2}+\frac{2 \mu \beta_{n} \lambda_{n+1}}{\lambda_{n+2}} \vartheta(1+\vartheta)\left\|x_{n+1}-x_{n}\right\|^{2} \\
& \quad-\beta_{n}\left(1-\frac{2 \mu \lambda_{n}}{\lambda_{n+1}}-\frac{2 \mu \lambda_{n+1}}{\lambda_{n+2}}(1+\vartheta)\right)\left\|w_{n}-y_{n}\right\|^{2} \\
& \quad-\beta_{n}\left(1-\frac{\mu \lambda_{n}}{\lambda_{n+1}}-\frac{2 \mu \lambda_{n+1}}{\lambda_{n+2}}(1+\vartheta)\right)\left\|z_{n}-y_{n}\right\|^{2} .
\end{aligned}
$$

By using Cauchy inequality, we have

$$
\begin{aligned}
& \left\|x_{n+1}-w_{n}\right\|^{2} \\
& =\left\|x_{n+1}-x_{n}-\vartheta_{n}\left(x_{n}-x_{n-1}\right)\right\|^{2} \\
& =\left\|x_{n+1}-x_{n}\right\|^{2}+\vartheta_{n}^{2}\left\|x_{n}-x_{n-1}\right\|^{2}-2 \vartheta_{n}\left\langle x_{n+1}-x_{n}, x_{n}-x_{n-1}\right\rangle \\
& \geq\left(1-\vartheta_{n}\right)\left\|x_{n+1}-x_{n}\right\|^{2}+\left(\vartheta_{n}^{2}-\vartheta_{n}\right)\left\|x_{n}-x_{n-1}\right\|^{2} .
\end{aligned}
$$

By definition of $x_{n+1}$, we have

$$
\left\|x_{n+1}-w_{n}\right\|^{2}=\beta_{n}^{2}\left\|z_{n}-w_{n}\right\|^{2} .
$$


Combining (15), (17) and (18), implies that

$$
\begin{aligned}
& \left\|x_{n+1}-p^{*}\right\|^{2}-\vartheta_{n+1}\left\|x_{n}-p^{*}\right\|^{2}+\frac{2 \mu \beta_{n+1} \lambda_{n+1}}{\lambda_{n+2}}\left\|w_{n+1}-y_{n}\right\|^{2} \\
& \leq\left\|x_{n}-p^{*}\right\|^{2}-\vartheta_{n}\left\|x_{n-1}-p^{*}\right\|^{2}+\frac{2 \mu \beta_{n} \lambda_{n}}{\lambda_{n+1}}\left\|w_{n}-y_{n-1}\right\|^{2} \\
& \quad+\vartheta(1+\vartheta)\left\|x_{n}-x_{n-1}\right\|^{2}+\frac{2 \mu \lambda_{n+1}}{\lambda_{n+2}} \vartheta(1+\vartheta)\left\|x_{n+1}-x_{n}\right\|^{2} \\
& \quad-\varrho_{n}\left[\left(1-\vartheta_{n}\right)\left\|x_{n+1}-x_{n}\right\|^{2}+\left(\vartheta_{n}^{2}-\vartheta_{n}\right)\left\|x_{n}-x_{n-1}\right\|^{2}\right],
\end{aligned}
$$

where $\varrho_{n}:=\frac{1}{2}\left(1-\frac{2 \mu \lambda_{n}}{\lambda_{n+1}}-\frac{2 \mu \lambda_{n+1}}{\lambda_{n+2}}(1+\vartheta)\right)$.

Let $\Psi_{n}:=\left\|x_{n}-p^{*}\right\|^{2}-\vartheta_{n}\left\|x_{n-1}-p^{*}\right\|^{2}+\frac{2 \mu \beta_{n} \lambda_{n}}{\lambda_{n+1}}\left\|w_{n}-y_{n-1}\right\|^{2}, Q_{n}:=$ $\varrho_{n}\left(1-\vartheta_{n}\right)-\frac{2 \mu \lambda_{n+1}}{\lambda_{n+2}} \vartheta(1+\vartheta)$ and $R_{n}:=\vartheta(1+\vartheta)+\varrho_{n} \vartheta_{n}\left(1-\vartheta_{n}\right)$. From the above substitutions the expression (19) turns into

$$
\Psi_{n+1} \leq \Psi_{n}+R_{n}\left\|x_{n}-x_{n-1}\right\|^{2}-Q_{n}\left\|x_{n+1}-x_{n}\right\|^{2} .
$$

Next, set $\Gamma_{n}:=\Psi_{n}+R_{n}\left\|x_{n}-x_{n-1}\right\|^{2}$ and with use (20), we obtain

$$
\begin{aligned}
\Gamma_{n+1}-\Gamma_{n} & =\Psi_{n+1}+R_{n+1}\left\|x_{n+1}-x_{n}\right\|^{2}-\Psi_{n}-R_{n}\left\|x_{n}-x_{n-1}\right\|^{2} \\
& \leq-Q_{n}\left\|x_{n+1}-x_{n}\right\|^{2}+R_{n+1}\left\|x_{n+1}-x_{n}\right\|^{2} \\
& =-\left(Q_{n}-R_{n+1}\right)\left\|x_{n+1}-x_{n}\right\|^{2}
\end{aligned}
$$

Next, we have to compute

$$
\begin{aligned}
Q_{n}-R_{n+1} \\
=\varrho_{n}\left(1-\vartheta_{n}\right)-\frac{2 \mu \lambda_{n+1}}{\lambda_{n+2}} \vartheta(1+\vartheta)-\vartheta(1+\vartheta)+\varrho_{n+1}\left(\vartheta^{2}-\vartheta\right) \\
\geq \varrho_{n}(1-\vartheta)-\frac{2 \mu \lambda_{n+1}}{\lambda_{n+2}} \vartheta(1+\vartheta)-\vartheta(1+\vartheta)+\varrho_{n+1}\left(\vartheta^{2}-\vartheta\right) \\
=\left(\frac{1}{2}-\frac{\mu \lambda_{n}}{\lambda_{n+1}}-\frac{\mu \lambda_{n+1}}{\lambda_{n+2}}-\frac{\mu \lambda_{n+1}}{\lambda_{n+2}} \vartheta\right)(1-\vartheta)-\frac{2 \mu \lambda_{n+1}}{\lambda_{n+2}}\left(\vartheta+\vartheta^{2}\right) \\
-\vartheta(1+\vartheta)+\left(\frac{1}{2}-\frac{\mu \lambda_{n+1}}{\lambda_{n+2}}-\frac{\mu \lambda_{n+2}}{\lambda_{n+3}}-\frac{\mu \lambda_{n+2}}{\lambda_{n+3}} \vartheta\right)\left(\vartheta^{2}-\vartheta\right) \\
=\left(\frac{1}{2}-2 \vartheta-\frac{1}{2} \vartheta^{2}\right)-\mu\left[\left(\frac{\lambda_{n}}{\lambda_{n+1}}+\frac{\lambda_{n+1}}{\lambda_{n+2}}\right)+\left(\frac{\lambda_{n+2}}{\lambda_{n+3}}\right) \vartheta^{3}\right. \\
+\left(\frac{\lambda_{n+1}}{\lambda_{n+2}}-\frac{\lambda_{n}}{\lambda_{n+1}}-\frac{\lambda_{n+1}}{\lambda_{n+2}}+\frac{2 \lambda_{n+1}}{\lambda_{n+2}}-\frac{\lambda_{n+1}}{\lambda_{n+2}}-\frac{\lambda_{n+2}}{\lambda_{n+3}}\right) \vartheta
\end{aligned}
$$




$$
\left.+\left(-\frac{\lambda_{n+1}}{\lambda_{n+2}}+\frac{2 \lambda_{n+1}}{\lambda_{n+2}}+\frac{\lambda_{n+1}}{\lambda_{n+2}}+\frac{\lambda_{n+2}}{\lambda_{n+3}}-\frac{\lambda_{n+2}}{\lambda_{n+3}}\right) \vartheta^{2}\right] .
$$

Due to $\lambda_{n} \rightarrow \lambda$ there exits a fixed number $\epsilon>0$ such that $\left(\forall n \geq N_{0}\right)$

$$
\epsilon \in\left(0, \frac{1}{2}-2 \vartheta-\frac{1}{2} \vartheta^{2}-\mu\left(2-\vartheta+2 \vartheta^{2}+\vartheta^{3}\right)\right) .
$$

The expression (22) gives that

$$
Q_{n}-R_{n+1} \geq \epsilon, \forall n \geq N_{0}
$$

Thus, expression (21) turn into

$$
\Gamma_{n+1}-\Gamma_{n} \leq-\epsilon\left\|x_{n+1}-x_{n}\right\|^{2} \leq 0
$$

Thus, $\left\{\Gamma_{n}\right\}$ is non-increasing. From $\Gamma_{n+1}$ for $n \geq N_{0}$, we have

$$
\Gamma_{n+1} \geq-\vartheta_{n+1}\left\|x_{n}-p^{*}\right\|^{2} .
$$

From $\Gamma_{n}$ for $n \geq N_{0}$, we get

$$
\Gamma_{n} \geq\left\|x_{n}-p^{*}\right\|^{2}-\vartheta_{n}\left\|x_{n-1}-p^{*}\right\|^{2} .
$$

Now, using (26) for $n \geq N_{0}$, we have

$$
\begin{aligned}
\left\|x_{n}-p^{*}\right\|^{2} & \leq \Gamma_{n}+\vartheta_{n}\left\|x_{n-1}-p^{*}\right\|^{2} \\
& \leq \Gamma_{N_{0}}+\vartheta\left\|x_{n-1}-p^{*}\right\|^{2} \\
& \leq \cdots \leq \Gamma_{N_{0}}\left(\vartheta^{n-N_{0}}+\cdots+1\right)+\vartheta^{n-N_{0}}\left\|x_{N_{0}}-p^{*}\right\|^{2} \\
& \leq \frac{\Gamma_{N_{0}}}{1-\vartheta}+\vartheta^{n-N_{0}}\left\|x_{N_{0}}-p^{*}\right\|^{2} .
\end{aligned}
$$

Combining (25) and (27), we obtain

$$
\begin{aligned}
-\Gamma_{n+1} & \leq \vartheta_{n+1}\left\|x_{n}-p^{*}\right\|^{2} \\
& \leq \vartheta\left\|x_{n}-p^{*}\right\|^{2} \\
& \leq \vartheta \frac{\Gamma_{N_{0}}}{1-\vartheta}+\vartheta^{n-N_{0}+1}\left\|x_{N_{0}}-p^{*}\right\|^{2} .
\end{aligned}
$$

It follows from (24) and (28) that

$$
\epsilon \sum_{n=N_{0}}^{k}\left\|x_{n+1}-x_{n}\right\|^{2} \leq \Gamma_{N_{0}}-\Gamma_{n+1}
$$




$$
\begin{aligned}
& \leq \Gamma_{N_{0}}+\vartheta \frac{\Gamma_{N_{0}}}{1-\vartheta}+\vartheta^{n-N_{0}+1}\left\|x_{N_{0}}-p^{*}\right\|^{2} \\
& \leq \frac{\Gamma_{N_{0}}}{1-\vartheta}+\left\|x_{N_{0}}-p^{*}\right\|^{2} .
\end{aligned}
$$

letting $k \rightarrow \infty$ in (29) we obtain

$$
\sum_{n=1}^{\infty}\left\|x_{n+1}-x_{n}\right\|<+\infty \text { implies that }\left\|x_{n+1}-x_{n}\right\| \rightarrow 0 \text { as } n \rightarrow \infty .
$$

From (16) and (30) such as

$$
\left\|x_{n+1}-w_{n}\right\| \rightarrow 0 \quad \text { as } \quad n \rightarrow \infty
$$

and

$$
0 \leq\left\|x_{n}-w_{n}\right\| \leq\left\|x_{n}-x_{n+1}\right\|+\left\|x_{n+1}-w_{n}\right\| \longrightarrow 0 \text { as } n \rightarrow \infty .
$$

From $\Psi_{n}$ and (28), we attain

$$
-\Psi_{n+1} \leq \vartheta \frac{\Gamma_{N_{0}}}{1-\vartheta}+\vartheta^{n-N_{0}+1}\left\|x_{N_{0}}-p^{*}\right\|^{2}+R_{n}\left\|x_{n}-x_{n-1}\right\|^{2} .
$$

By expression (15) we can rewrite as

$$
\begin{aligned}
& \beta\left(1-\frac{2 \mu \lambda_{n}}{\lambda_{n+1}}-\frac{2 \mu \lambda_{n+1}}{\lambda_{n+2}}(1+\vartheta)\right)\left[\left\|w_{n}-y_{n}\right\|^{2}+\left\|z_{n}-y_{n}\right\|^{2}\right] \\
& \leq \Psi_{n}-\Psi_{n+1}+\vartheta(1+\vartheta)\left\|x_{n}-x_{n-1}\right\|^{2}+\frac{2 \mu \lambda_{0}}{\lambda} \vartheta(1+\vartheta)\left\|x_{n+1}-x_{n}\right\|^{2} .
\end{aligned}
$$

Summing up them (34) for $k \geq N_{0}$, we obtain

$$
\begin{aligned}
& \beta\left(1-\frac{2 \mu \lambda_{n}}{\lambda_{n+1}}-\frac{2 \mu \lambda_{n+1}}{\lambda_{n+2}}(1+\vartheta)\right)\left[\left\|w_{n}-y_{n}\right\|^{2}+\left\|z_{n}-y_{n}\right\|^{2}\right] \\
& \leq \Psi_{N_{0}}-\Psi_{k+1}+\vartheta(1+\vartheta) \sum_{n=N_{0}}^{k}\left\|x_{n}-x_{n-1}\right\|^{2} \\
& \quad+\frac{2 \mu \lambda_{0}}{\lambda} \vartheta(1+\vartheta) \sum_{n=N_{0}}^{k}\left\|x_{n+1}-x_{n}\right\|^{2} \\
& \leq \Psi_{N_{0}}+\vartheta \frac{\Gamma_{N_{0}}}{1-\vartheta}+\vartheta^{n-N_{0}+1}\left\|x_{N_{0}}-p^{*}\right\|^{2}+R\left\|x_{k}-x_{k-1}\right\|^{2}
\end{aligned}
$$




$$
+\left(\vartheta+\vartheta^{2}\right) \sum_{n=N_{0}}^{k}\left\|x_{n}-x_{n-1}\right\|^{2}+\frac{2 \mu \lambda_{0}}{\lambda}\left(\vartheta+\vartheta^{2}\right) \sum_{n=N_{0}}^{k}\left\|x_{n+1}-x_{n}\right\|^{2} .
$$

where $R:=\vartheta(1+\vartheta)+\frac{1}{2}(1-\vartheta)$ and taking $k \rightarrow \infty$, we have

$$
\sum_{n}\left\|w_{n}-y_{n}\right\|^{2}=\sum_{n}\left\|z_{n}-y_{n}\right\|^{2}<+\infty
$$

and

$$
\lim _{n \rightarrow \infty}\left\|w_{n}-y_{n}\right\|=\lim _{n \rightarrow \infty}\left\|z_{n}-y_{n}\right\|=0 .
$$

By (13), (30), (31) and (37), we deduce the followings:

$$
\begin{gathered}
\lim _{n \rightarrow \infty}\left\|x_{n+1}-y_{n}\right\|=\lim _{n \rightarrow \infty}\left\|x_{n}-y_{n}\right\|=0 . \\
\lim _{n \rightarrow \infty}\left\|w_{n}-y_{n-1}\right\|=\lim _{n \rightarrow \infty}\left\|y_{n}-y_{n-1}\right\|=0 .
\end{gathered}
$$

By letting $k \rightarrow \infty$ in (14) through (30), (36) implies that

$$
\sum_{n}\left\|w_{n+1}-y_{n}\right\|^{2}<\infty
$$

The expression (10) and (11) with Lemma 2.3, provides that limit of $\left\|x_{n}-p^{*}\right\|$ exists. The sequences $\left\{x_{n}\right\},\left\{w_{n}\right\}$ and $\left\{y_{n}\right\}$ are bounded. Now, we prove that every sequential weak limit point of the sequence $\left\{x_{n}\right\}$ is in $E P(f, \mathbb{K})$. Let $z$ is a weak limit point of $\left\{x_{n}\right\}$, i.e. there is a subsequence, represent through $\left\{x_{n_{k}}\right\}$ of $\left\{x_{n}\right\}$ converges weakly to $z$. Thus, $\left\{y_{n_{k}}\right\}$ also converges weakly to $z \in \mathbb{K}$. Assume that that $z \in E P(f, \mathbb{K})$. By (3), (4) and the definition of $\lambda_{n+1}$, we have

$$
\begin{aligned}
& \lambda_{n_{k}} f\left(y_{n_{k}}, y\right) \\
& \geq \lambda_{n_{k}} f\left(y_{n_{k}}, z_{n_{k}}\right)+\left\langle w_{n_{k}}-z_{n_{k}}, y-z_{n_{k}}\right\rangle \\
& \geq \lambda_{n_{k}} f\left(y_{n_{k}-1}, x_{n_{k+1}}\right)-\lambda_{n_{k}} f\left(y_{n_{k}-1}, y_{n_{k}}\right)-\frac{\mu \lambda_{n_{k}}}{2 \lambda_{n_{k}+1}}\left\|y_{n_{k}}-y_{n_{k}-1}\right\|^{2} \\
& \quad-\frac{\mu \lambda_{n_{k}}}{2 \lambda_{n_{k}+1}}\left\|y_{n_{k}}-z_{n_{k}}\right\|^{2}+\left\langle w_{n_{k}}-z_{n_{k}}, y-z_{n_{k}}\right\rangle \\
& \geq\left\langle w_{n_{k}}-y_{n_{k}}, z_{n_{k}}-y_{n_{k}}\right\rangle-\frac{\mu \lambda_{n_{k}}}{2 \lambda_{n_{k}+1}}\left\|y_{n_{k}}-y_{n_{k}-1}\right\|^{2} \\
& \quad-\frac{\mu \lambda_{n_{k}}}{2 \lambda_{n_{k}+1}}\left\|y_{n_{k}}-z_{n_{k}}\right\|^{2}+\left\langle w_{n_{k}}-z_{n_{k}}, y-z_{n_{k}}\right\rangle
\end{aligned}
$$


where $y \in E_{n}$. From (37), (38), (39) and the boundedness of $\left\{x_{n}\right\}$ that the right-hand side of the above inequality goes to zero. By $\lambda_{n_{k}} \geq \lambda>0$, we have

$$
0 \leq \limsup _{k \rightarrow \infty} f\left(y_{n_{k}}, y\right) \leq f(z, y), \forall y \in E_{n}
$$

Due to $\mathbb{K} \subset E_{n}$ and $f(z, y) \geq 0$, for all $y \in \mathbb{K}$. This proved $z \in E P(f, \mathbb{K})$. By Lemma ??, ensures that $\left\{w_{n}\right\},\left\{x_{n}\right\}$ and $\left\{y_{n}\right\}$ weakly converges to $p^{*}$ as $n \rightarrow \infty$.

\section{Application to variational inequality problem}

In this section we discuss the application of Theorem 3.1 to solve a pseudomonotone variational inequality problems with Lipschitz-type continuous operator. An operator $F: \mathbb{K} \rightarrow \mathbb{E}$ is said to be

(F1) pseudomonotone on $\mathbb{K}$ if

$$
\left\langle F\left(x_{1}\right), x_{2}-x_{1}\right\rangle \geq 0 \Longrightarrow\left\langle F\left(x_{2}\right), x_{1}-x_{2}\right\rangle \leq 0, \forall x_{1}, x_{2} \in \mathbb{K}
$$

(F2) L-Lipschitz continuous on $\mathbb{K}$ if

$$
\left\|F\left(x_{1}\right)-F\left(x_{2}\right)\right\| \leq L\left\|x_{1}-x_{2}\right\|, \forall x_{1}, x_{2} \in \mathbb{K}
$$

The variational inequality problem is described as follows:

$$
p^{*} \in \mathbb{K} \text { such that }\left\langle F\left(p^{*}\right), y-p^{*}\right\rangle \geq 0, \forall y \in \mathbb{K} \text {. }
$$

Note: If $f(x, y):=\langle F(x), y-x\rangle$ for all $x, y \in \mathbb{K}$, then problem (EP) turns to (VIP) with $L=2 c_{1}=2 c_{2}$.

Corollary 4.1. Assume that $F: \mathbb{K} \rightarrow \mathbb{E}$ satisfies the conditions (F1)-(F2). Let $\left\{x_{n}\right\}$ be the sequence generated as follows:

(i) Choose $x_{-1}, x_{0}, y_{0} \in \mathbb{E}, \lambda_{0}=\lambda_{1}>0$ and $0 \leq \vartheta_{n} \leq \vartheta<\sqrt{5}-2$ is non-decreasing. Set

$$
x_{1}=P_{\mathbb{K}}\left(w_{0}-\lambda_{0} F y_{0}\right), \quad y_{1}=P_{\mathbb{K}}\left(w_{1}-\lambda_{1} F y_{0}\right),
$$

where $w_{0}=x_{0}+\vartheta_{0}\left(x_{0}-x_{-1}\right)$ and $w_{1}=x_{1}+\vartheta_{1}\left(x_{1}-x_{0}\right)$. 
(ii) Given $x_{n-1}, x_{n}, y_{n-1}, y_{n}$ for $n \geq 1$. Compute

$$
x_{n+1}=\left(1-\beta_{n}\right) w_{n}+\beta_{n} z_{n} \text { and } z_{n}=P_{E_{n}}\left(w_{n}-\lambda_{n} F y_{n}\right) \text {, }
$$

where $w_{n}=x_{n}+\vartheta_{n}\left(x_{n}-x_{n-1}\right), 0<\beta \leq \beta_{n} \leq 1$ and

$$
E_{n}=\left\{z \in \mathbb{E}:\left\langle w_{n}-\lambda_{n} F w_{n}-y_{n}, z-y_{n}\right\rangle \leq 0\right\}
$$

(iii) Compute

$$
y_{n+1}=P_{\mathbb{K}}\left(w_{n+1}-\lambda_{n+1} F y_{n}\right)
$$

where $w_{n+1}=x_{n+1}+\vartheta_{n+1}\left(x_{n+1}-x_{n}\right),[t]_{+}=\max \{t, 0\}$ and

$$
\lambda_{n+1}=\min \left\{\lambda_{n}, \frac{\mu\left(\left\|y_{n-1}-y_{n}\right\|^{2}+\left\|z_{n}-y_{n}\right\|^{2}\right)}{2\left[\left\langle F\left(y_{n-1}\right)-F\left(y_{n}\right), z_{n}-y_{n}\right\rangle\right]_{+}}\right\} .
$$

Moreover, we have control parameters conditions, i.e.,

$$
0<\mu<\frac{\frac{1}{2}-2 \vartheta-\frac{1}{2} \vartheta^{2}}{2-\vartheta+2 \vartheta^{2}+\vartheta^{3}} \quad \text { with } \quad 0 \leq \vartheta_{n} \leq \vartheta<\sqrt{5}-2 .
$$

Then, the sequence $\left\{x_{n}\right\}$ weakly converges $p^{*}$ of $(V I P)$ on $\mathbb{K}$.

\section{Numerical illustration}

Numerical results are discussed in this section to illustrate the efficiency of our proposed methodology. The MATLAB code is being used in MATLAB edition 9.5 (R2018b) on the Intel(R) Core(TM)i5-6200 Processor PC @ 2.30GHz 2.40GHz, RAM 8.00 GB.

Example 5.1. Consider the Nash-Cournot oligopolistic equilibrium model [41]. Assume that there are $n$ firms that assemble the same product. Let $x$ serve as a vector where each component $x_{i}$ represent the quantity of the product made by the firm $i$. The value function $P$ for each individual firm is represented as $P_{i}(S)=\phi_{i}-\psi_{i} S$, where $\phi_{i}>0, \psi_{i}>0$ and $S=\sum_{i=1}^{m} x_{i}$. The profit function $F_{i}(x)=P_{i}(S) x_{i}-t_{i}\left(x_{i}\right)$, while $t_{i}\left(x_{i}\right)$ is the import duty and payment for generating $x_{i}$. The design scheme for the entire theory is getting the set of $\mathbb{K}:=\mathbb{K}_{1} \times \mathbb{K}_{2} \times \cdots \times \mathbb{K}_{n}$, where $\mathbb{K}_{i}=\left[x_{i}^{\min }, x_{i}^{\max }\right]$. Each firm seeks to carry out its optimum contribute by going into account the following amount of demand 
on the assumption that the output of all the other firms would be an input parameter. A point $p^{*} \in \mathbb{K}=\mathbb{K}_{1} \times \mathbb{K}_{2} \times \cdots \times \mathbb{K}_{n}$ is an equilibrium position of the model if

$$
F_{i}\left(p^{*}\right) \geq F_{i}\left(p^{*}\left[x_{i}\right]\right), \forall x_{i} \in \mathbb{K}_{i}, \forall i=1,2, \cdots, n
$$

where $p^{*}\left[x_{i}\right]$ serve as the vector from $p^{*}$ by receiving $x_{i}^{*}$ with $x_{i}$. Let $f(x, y):=$ $\varphi(x, y)-\varphi(x, x)$ with $\varphi(x, y):=-\sum_{i=1}^{n} F_{i}\left(x\left[y_{i}\right]\right)$, and the complication of getting the Nash equilibrium point is

$$
\text { Find } p^{*} \in \mathbb{K}: f\left(p^{*}, y\right) \geq 0, \forall y \in \mathbb{K} \text {. }
$$

The bifunction $f$ can be used in the following form.

$$
f(x, y)=\langle P x+Q y+q, y-x\rangle
$$

where $q \in \mathbb{R}^{5}$ and $A, B$ are

$$
P=\left(\begin{array}{ccccc}
3.1 & 2 & 0 & 0 & 0 \\
2 & 3.6 & 0 & 0 & 0 \\
0 & 0 & 3.5 & 2 & 0 \\
0 & 0 & 2 & 3.3 & 0 \\
0 & 0 & 0 & 0 & 3
\end{array}\right) \quad Q=\left(\begin{array}{ccccc}
1.6 & 1 & 0 & 0 & 0 \\
1 & 1.6 & 0 & 0 & 0 \\
0 & 0 & 1.5 & 1 & 0 \\
0 & 0 & 1 & 1.5 & 0 \\
0 & 0 & 0 & 0 & 2
\end{array}\right)
$$

$q=(1,-2,-1,2,-1)^{T}$ while Lipschitz constants are $c_{1}=c_{2}=\frac{1}{2}\|P-Q\|$ (see $[6])$. The feasible set $\mathbb{K} \subset \mathbb{R}^{n}$ is $\mathbb{K}:=\left\{x \in \mathbb{R}^{5}:-2 \leq x_{i} \leq 5\right\}$. The numerical findings are shown in the Figure $1-2$ and Table 1 . We use $x_{-1}=x_{0}=y_{0}=$ $(1,1,1,1,1)^{T}$ and consider $\vartheta=\vartheta_{n}=0.12$ gives $\mu=0.12<0.1323$ from the given formula. 


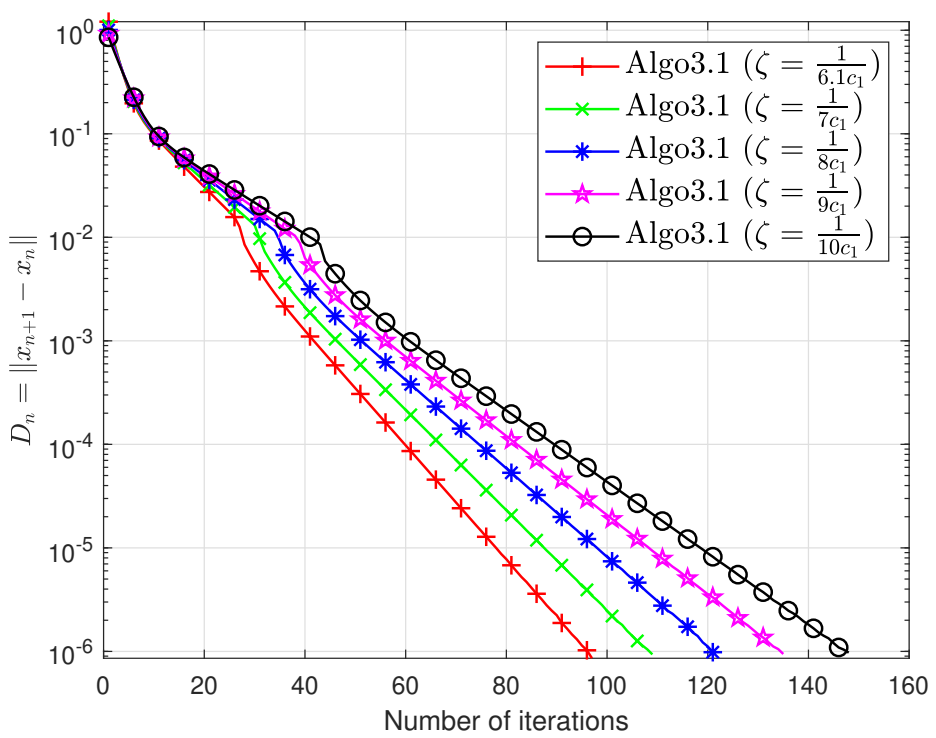

Figure 1: Algorithm in [33] for different values of $\lambda$.

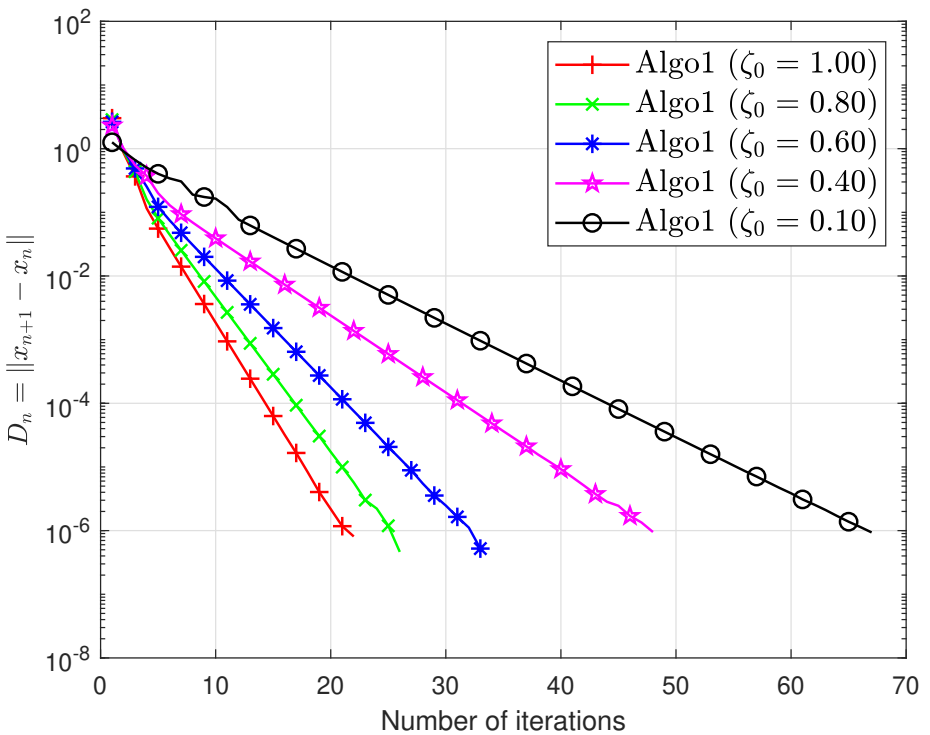

Figure 2: Algorithm 1 behaviour for values of $\lambda_{0}$. 
Table 1: Algorithm 1 (Algo1) and Algorithm 3. 1 (Algo3.1) in [33].

\begin{tabular}{l|c|c|c|c|c|cccc}
\hline & & & & & & \multicolumn{2}{c}{ Algo3.1 } & & \multicolumn{2}{c}{ Algo1 } \\
\cline { 9 - 10 }$\lambda_{0}$ & $\lambda$ & $\vartheta_{n}$ & $\beta_{n}$ & TOL & No. Iter. & CPU time(s) & & No. Iter. & CPU time(s) \\
\hline \hline 1 & $\frac{1}{6.1 c_{1}}$ & 0.12 & 0.80 & $10^{-6}$ & 97 & 2.0117 & & 22 & 0.6898 \\
0.8 & $\frac{1}{7 c_{1}}$ & 0.12 & 0.80 & $10^{-6}$ & 109 & 2.3656 & & 26 & 0.7891 \\
0.6 & $\frac{1}{7.5 c_{1}}$ & 0.12 & 0.80 & $10^{-6}$ & 121 & 3.1567 & & 33 & 0.9872 \\
0.4 & $\frac{1}{8 c_{1}}$ & 0.12 & 0.80 & $10^{-6}$ & 135 & 3.4834 & & 48 & 1.1243 \\
0.1 & $\frac{1}{9 c_{1}}$ & 0.12 & 0.80 & $10^{-6}$ & 148 & 3.5834 & & 67 & 1.4356 \\
\hline
\end{tabular}

\section{Discussion on numerical experiments:}

(i) No previous knowledge on Lipschitz-constant $c_{1}, c_{2}$ is required to run the Algorithm 1.

(ii) In the Algorithm 1, stepsize is independent of the Lipschitz-constant choice and uses an explicit stepsize evaluation formula based on previous iterations.

(iii) We can see that Algorithm in [33] is perform better when the stepsize value is close to the $\frac{1}{2 c_{2}+4 c_{1}}$.

(iv) We can see that Algorithm 1 works better when $\lambda_{0}$ had value close to 1 .

(v) We can see that Algorithm 1 works far better when $\vartheta_{n}$ is close to $\sqrt{5}-2$.

\section{Conclusion}

This article suggests new algorithms to solve the problems of pseudomonotone equilibrium. The basic edge of this algorithm is that the step-size, in this case, is independent of the Lipschitz constant type choice. The reasoning is that we are using an explicit step-by-step evaluation procedure. Numerical experiments have also been reported to see the performance of our porposed method, and we can see that the inertial factor is usually performing much better.

\section{Acknowledgements}

Nopparat Wairojjana was partially supported by Valaya Alongkorn Rajabhat University under the Royal Patronage, Thailand. Tiwabhorn Khanpanuk would like to thanks Phetchabun Rajabhat University, Thailand. 


\section{References}

[1] E. Blum, From optimization and variational inequalities to equilibrium problems, Math. Student, 63 (1994), 123-145.

[2] F. Facchinei, J.S. Pang, Finite-dimensional Variational inequalities and Complementarity Problems, Springer Science, New York (2007).

[3] I. Konnov, Equilibrium Models and Variational Inequalities, Elsevier, New York (2007).

[4] L.D. Muu, W. Oettli, Convergence of an adaptive penalty scheme for finding constrained equilibria, Nonlinear Anal., 18, No 12 (1992), 1159-1166.

[5] K. Fan, A minimax Inequality and Applications, Inequalities III (O. Shisha, Ed.), Academic Press, New York (1972).

[6] T.D. Quoc, L.D. Muu, V.H. Nguyen, Extragradient algorithms extended to equilibrium problems, Optim., 57, (2008), 749-776.

[7] T.D. Quoc, P.N. Anh, L.D. Muu, Dual extragradient algorithms extended to equilibrium problems, J. Global Optim., 52 (2011), 139-159.

[8] S.I. Lyashko, V.V. Semenov, A new two-step proximal algorithm of solving the problem of equilibrium programming, In: Optimization and Its Applications in Control and Data Sciences, Springer Intern. Publ., Switzerland (2016), 315-325.

[9] H.U. Rehman, P. Kumam, Y.J. Cho, Y.I. Suleiman, W. Kumam, Modified Popov's explicit iterative algorithms for solving pseudomonotone equilibrium problems, Optim. Methods Softw., (2020), 1-32; doi:10.1080/10556788.2020.1734805.

[10] S. Takahashi, W. Takahashi, Viscosity approximation methods for equilibrium problems and fixed point problems in Hilbert spaces, J. Math. Anal. Appl., 331 (2007), 506-515.

[11] H.U. Rehman, N. Pakkaranang, A. Hussain, N. Wairojjana, A modified extra-gradient method for a family of strongly pseudomonotone equilibrium problems in real Hilbert spaces, J. Math. Comput. Sci., 22 (2020), $38-48$. 
[12] N. Wairojjana, H.U. Rehman, I.K. Argyros, N. Pakkaranang, An accelerated extragradient method for solving pseudomonotone equilibrium problems with applications, Axioms, 9 (2020), \# 99; doi:10.3390/axioms9030099.

[13] N. Wairojjana, H.U. Rehman, M.D. la Sen, N. akkaranang, A general inertial projection-type algorithm for solving equilibrium problem in Hilbert spaces with applications in fixed-point problems, Axioms, 9 (2020), \# 101; doi:10.3390/axioms9030101.

[14] P.N. Anh, T.N. Hai, P.M. Tuan, On ergodic algorithms for equilibrium problems, J. Glob. Optim., 64 (2015), 179-195.

[15] D.V. Hieu, P.K. Quy, L.V. Vy, Explicit iterative algorithms for solving equilibrium problems, Calcolo, 56 (2019); doi:10.1007/s10092-019-0308-5.

[16] D.V. Hieu, New extragradient method for a class of equilibrium problems in Hilbert spaces, Appl. Anal., 97 (2017), 811-824.

[17] P. Santos, S. Scheimberg, An inexact subgradient algorithm for equilibrium problems, Comput. Appl. Math., 30 (2011), 91-107.

[18] H.U. Rehman, P. Kumam, A.B. Abubakar, Y.J. Cho, The extragradient algorithm with inertial effects extended to equilibrium problems, Comput. Appl. Math., 39 (2020); doi:10.1007/s40314-020-1093-0.

[19] H.U. Rehman, P. Kumam, W. Kumam, M. Shutaywi, W. Jirakitpuwapat, The inertial sub-gradient extra-gradient method for a class of pseudomonotone equilibrium problems, Symmetry, 12 (2020), \# 63.

[20] P.N. Anh, L.T. Anh, The subgradient extragradient method extended to equilibrium problems, Opti., 64 (2010), 225-248.

[21] H.U. Rehman, P. Kumam, I.K. Argyros, W. Deebani, W. Kumam, Inertial extra-gradient method for solving a family of strongly pseudomonotone equilibrium problems in real Hilbert spaces with application in variational inequality problem, Symmetry, 12 (2020), \# 503.

[22] L.D. Muu, T.D. Quoc, Regularization algorithms for solving monotone Ky fan inequalities with application to a nash-cournot equilibrium model, J. Optim. Theory Appl., 142 (2019), 185-204. 
[23] H.U. Rehman, P. Kumam, I.K. Argyros, N.A. Alreshidi, W. Kumam, W. irakitpuwapat, A self-adaptive extra-gradient methods for a family of pseudomonotone equilibrium programming with application in different classes of variational inequality problems, Symmetry, 12 (2020), \# 523.

[24] H.U. Rehman, P. Kumam, I.K. Argyros, M. Shutaywi, Z. Shah, Optimization based methods for solving the equilibrium problems with applications in variational inequality problems and solution of nash equilibrium models, Math., 8 (2020), \# 822.

[25] H.U. Rehman, P. Kumam, M. Shutaywi, N.A. Alreshidi, W. Kumam, Inertial optimization based two-step methods for solving equilibrium problems with applications in variational inequality problems and growth control equilibrium models, Energ., 13 (2020), \# 3292.

[26] P. Yordsorn, P. Kumam, H.U. Rehman, A.H. Ibrahim, A weak convergence self-adaptive method for solving pseudomonotone equilibrium problems in a real Hilbert space, Math., 8 (2020), \# 1165.

[27] B. Martinet, Régularisation d'inéquations variationnelles par approximations successives, Rev. Française Informat. Recherche Opérationnelle, 4 (1970), 154-158.

[28] R.T. Rockafellar, Monotone operators and the proximal point algorithm, SIAM J. Control Optim., 14 (1976), 877-898.

[29] A. Moudafi, Proximal point algorithm extended to equilibrium problems, J. Nat. Geom., 15 (1999), 91-100.

[30] G. Cohen, Auxiliary problem principle and decomposition of optimization problems, J. Optim. Theory Appl., 32 (1980), 277-305.

[31] G. Cohen, Auxiliary problem principle extended to variational inequalities, J. Optim. Theory Appl., 59 (1988), 325-333.

[32] G. Mastroeni, On auxiliary principle for equilibrium problems, In: Equilibrium Problems and Variational Models, Springer, New York (2003), 289298.

[33] Y. Liu, H. Kong, The new extragradient method extended to equilibrium problems, Rev. R. Acad. Cienc. Exactas Fús. Nat. Ser. A Mat., 113 (2018), 2113-2126. 
[34] L.D. Popov, A modification of the Arrow-Hurwicz method for search of saddle points, USSR Comput. Math. Math. Phys., 28 (1980), 845-848; doi:10.1007/BF01141092.

[35] B.T. Polyak, Some methods of speeding up the convergence of iteration methods, USSR Comput. Math. Math. Phys., 4 (1964), 1-17.

[36] M. Bianchi, S. Schaible, Generalized monotone bifunctions and equilibrium problems, J. Optim. Theory Appl., 90 (1996), 31-43.

[37] J.V. Tiel, Convex Analysis: An Introductory Text, Wiley, New York (1984).

[38] H.H. Bauschke, P.L. Combettes, Convex Analysis and Monotone Operator Theory in Hilbert Spaces, Springer, New York (2011).

[39] F. Alvarez, H. Attouch, An inertial proximal method for maximal monotone operators via discretization of a nonlinear oscillator with damping, Set-Valued Var. Anal., 9 (2001), 3-11; doi:10.1023/A:1011253113155.

[40] Z. Opial, Weak convergence of the sequence of successive approximations for nonexpansive mappings, Bull. Amer. Math. Soc., 73 (1967), 591-598.

[41] N. Quy, V. Nguyen, L. Muu, On the cournot-nash oligopolistic market equilibrium models with concave cost functions, J. Global Optim., 41 (2005), 351-364. 
